\title{
PLANTEAMIENTOS ANTE LA INTERSUBJETIVIDAD: EL SYMPATHIEBUCH DE MAX SCHELER Y SU REPER- CUSIÓN EN SER Y TIEMPO DE MARTIN HEIDEGGER ${ }^{1}$
}

Considerations about the intersubjectivity: The Sympathiebuch of

Max Scheler and his repercussion in Being and Time by Martin Heidegger

Enrique Muñoz Pérez*

Resumen

Este estudio pretende clarificar influencias efectivas de la reflexión de Scheler en el pensamiento de Heidegger para lo cual el autor se concentra en los principales análisis de Scheler en el Sympathiebuch, y en especial de su Anexo, que influyen poderosamente en la teoría sobre la intersubjetividad que bosqueja Heidegger en Ser y Tiempo.

Palabras clave: Empatía, inferencia de analogía, el otro, comunidad, uno.

Abstract

This study tries to clarify some effective influences of the Scheler's philosophy in the Heidegger's thought. I center, then, in Scheler's principal analyses in the Sympathiebuch, especially of his appendix, which they influence powerfully in the theory on the intersubjectivity that Heidegger sketches in Being and Time.

Key words: Empathy, deduction of analogy, the other, community, self (man).

INTRODUCCIÓN

En otra ocasión, me referí a la, a mi juicio, desatendida relación entre Martin Heidegger (1889-1976) y Max Scheler (1874-1928). Allí mostré algunas de las referencias mutuas, lecturas e interpretaciones que ambos filósofos llevan a cabo, en especial a fines de la década de los años veinte, período que corresponde a la época tardía del pensamiento de Scheler, dada su prematura muerte, y al entorno de Ser y Tiempo (1927) la obra principal de Heidegger. Más allá de los datos anecdóticos y de las referencias cruzadas entre ambos filósofos, pretendí iniciar un programa de trabajo en el que fuese posible mostrar las influencias filosóficas tanto explícitas como implícitas entre Scheler y Heidegger. ${ }^{2}$

\footnotetext{
${ }^{1}$ Trabajo escrito en el marco del Proyecto de Investigación Postdoctoral Fondecyt N ${ }^{\circ}$ 3085016: "Una relación olvidada: La influencia del pensamiento filosófico de Max Scheler en la concepción ontológica del hombre de Martin Heidegger (2007-2009)" / Agradezco al Profesor Dr. Alejandro G. Vigo por sus detalladas observaciones y comentarios, efectuados en una pasantía que realicé en la Universidad de Navarra (España). También doy las gracias a mi amigo y colega Profesor Dr. Jorge Alarcón L. por la lectura detenida del texto y sus agudos comentarios.

${ }^{2}$ Cfr. E. Muñoz. "Una relación olvidada: Heidegger y Scheler", en Revista de Filosofía, Universidad de Chile, Vol. 65 (2009):177-188.
} 
Pretendo, aquí, desarrollar un punto más bien sistemático de la reflexión que desarrolla Scheler sobre la intersubjetividad a comienzos de la segunda década del siglo XX y la resonancia que tiene en Heidegger, fundamentalmente en Ser y Tiempo. Para ello, comentaré algunos puntos de una obra poco conocida de Scheler denominada Zur Phänomenologie und Theorie der Sympathiegefühle und von Liebe und Hass (1913), que tiene un anexo muy relevante titulado: "Über den Grund zur Annahme der Existenz des fremden Ich". ${ }^{3}$ En otras palabras, la hipótesis que quiero sostener es que el Sympathiebuch, específicamente, su anexo, ejerce una destacada influencia en el pensamiento de Heidegger, en especial, en la concepción sobre la intersubjetividad que éste bosqueja en Ser y Tiempo. De este modo, el presente artículo comprende: (1) Antecedentes histórico-críticos del problema, (2) Comentario del Anexo del Sympathiebuch y (3) Alcances a Ser y Tiempo.

\section{ANTECEDENTES HISTÓRICO-CRÍTICOS DEL PROBLEMA}

La perspectiva que orienta el presente trabajo no es producto de una mera especulación, sino que se encuentra sustentada en afirmaciones del mismo Heidegger, quien a lo menos en cuatro lugares distintos de su obra se refiere al Sympathiebuch. Si bien estas referencias no son detalladas, permiten al menos identificar el impacto que la obra de Scheler tuvo en Heidegger ${ }^{4}$ y, al mismo tiempo, determinar las problemáticas del Sympathiebuch que más interesaron al filósofo de Messkirch. No seguiré necesariamente un orden cronológico en la presentación de las citas, sino que más bien problemático. De este modo, las referencias son las siguientes:

1.1. En la lección magistral denominada Grundprobleme der Phänomenologie (semestre de invierno 1919/20), específicamente en el "anexo A" (Anhang A), "Puntos a considerar para la reformulación" (Merkpunkte für die Umarbeitung), afirma Heidegger

Junto a la idea fenomenológica de la experiencia de la vida fáctica se debe estudiar su totalidad (en el todo de una indivisibilidad), también en relación con el mundo propio, también para la significatividad (compuesto mixto) [Scheler]1; además, lo particular de la "comunidad de vida".

Coejecución en la significatividad específica de los actos vivientes, "con" carácter de la significatividad; no empero basándose en la comunicación, en la cual el "con" se daría en una circunstancia fenomenal. (Scheler, Sympathiegehfühle) (Heidegger, 1993:197). ${ }^{5}$

\footnotetext{
${ }^{3}$ En adelante será citado como Sympathiebuch. Para éste $-\mathrm{y}$ para los otros títulos a que haremos referencia no hay traducción oficial al español— de manera que remitiremos a las denominaciones originales.

${ }^{4}$ Tal parece que Heidegger no es el único pensador que se ve impactado por el texto de Scheler. Karl Jaspers, por ejemplo, lo cita en Psychologie der Weltanschauung (1954:123), también comentado por Heidegger.

5 "Bei der phänomenologischen Anschauung der faktischen Lebenserfahrung ihre Totalität (im Ganzen einer Ungegliedertheit) auch bezüglich der Selbstwelt zu studieren - auch für Bedeutsamkeit (compositum mixtum) [Scheler]1; ferner das Eigentümliche der "Lebensgemeinschaft". Mitvollzug der in der Bedeutsamkeit spezifisch lebenden Akte - "Mit" -Charakter der Bedeutsamkeit; nicht aber auf Mitteilung 
Más allá de las observaciones y mejoras de Heidegger sobre su propio trabajo, que no es del caso detallar, he incluido en la cita los números de las notas a pie de página del texto de Heidegger porque las dos primeras remiten a $E l$ formalismo en la ética y la ética material de los valores (1913) — en adelante lo citaremos como Ética - de Max Scheler y la tercera al Sympathiebuch.

1.2. Unos años más tarde, en la lección magistral llamada Fenomenología de la vida religiosa (semestre de invierno 1920/1 semestre de verano 1921) y, específicamente, en el marco de la explicación fenomenológica de la Primera Epístola de San Pablo a los Tesalonicenses (\$23), ${ }^{6}$ Heidegger enfrenta algunas dificultades metodológicas, por lo que se pregunta cómo ponerse en el lugar de San Pablo, puesto que nosotros - sostieneaparentemente, no conocemos nada de su entorno. Sostiene Heidegger

Hay que recordar una dificultad metodológicamente más. Podría decirse que es imposible o sólo posible con restricciones colocarse en la situación exacta de San Pablo. Pues no conocemos su mundo circundante. Esta objeción brota de la concepción de que lo objetual dado previamente es primordial para una situación en la que hay que "introyectarse anímicamente/empatizar" (einfühlen) [E. Muñoz]. Pero hay que juzgar la posición con respecto al entorno de San Pablo a partir de su personalidad y preguntarse si el entorno es relevante para él. El problema de la "endopatía/empatía" (Einfühlung) [E. Muñoz] se plantea gnoseológicamente y por ello es errado en su principio. La concepción de Scheler se acerca al máximo, si bien está en gran medida gravada por su teoría del conocimiento (Heidegger, 2006:114).

En esta cita, emerge uno los problemas que trata, como expondré más adelante, el Sympathiebuch de Scheler, esto es, el problema de la "empatía" (Einfühlung). Si bien Heidegger valora el tratamiento del tema desarrollado por Scheler, considera que se encuentra lastrado por la perspectiva gnoseológica o de la teoría del conocimiento.

1.3. Zur Sache des Denkens. En este texto, aparecido para honrar al editor alemán Hermann Niemeyer, específicamente en el apartado denominado Mein Weg in die Phänomenologie (1963), Heidegger recuerda que "en el mismo año 1913 apareció

beruhend, in dem das "Mit” zur phänomenalen Gegebenheit käme (Scheler, Sympathiegehfühle) (Heidegger, 1993:197). La gran mayoría de las traducciones son mías, salvo que cite los textos disponibles en español. He privilegiado el sentido de lo que el autor quiere expresar, más que la literalidad. Por ello, he incorporado los textos en su idioma original, de manera tal de ofrecerle al lector la posibilidad de compararlos.

${ }^{6}$ En español esta lección magistral se encuentra en el texto Introducción a la fenomenología de la religión (2006).

${ }^{7}$ Sin ánimo de detenerme en detalles filológicos, no estoy de acuerdo con la traducción que hace Jorge Uscatescu del término "einfühlen" como "introyectarse anímicamente". Me parece más razonable la opción que toma Jorge E. Rivera, quien lo traduce como "empatizar". En las notas a su traducción de Ser y Tiempo (149), Rivera sostiene que "la palabra alemana Einfühlung dice, literalmente, un sentir que penetra en el otro, de tal manera que lo que yo siento en mí me ayuda para saber lo que siente el otro. Obviamente éste no es un término feliz" (Heidegger, 1997:473). 
en la editorial Max Niemeyer la significativa investigación de Max Scheler Zur Phänomenologie der Sympathiegefühle und von Liebe und Hass. Con un anexo Über den Grund zur Annahme der Existenz des fremden Ich (2000:85). ${ }^{8}$ Heidegger quiere destacar, con esta y otras referencias, el lugar privilegiado que dio la mencionada editorial a la investigación fenomenológica que emergió como una nueva perspectiva de la filosofía alemana.

1.4. Ser y Tiempo. La alusión al texto de Scheler se encuentra en una nota del parágrafo §25 de Ser y Tiempo (1927). En el contexto de una de sus principales conclusiones preliminares - esto es, que no es posible pensar un sujeto aislado del mundo- Heidegger alude a la necesidad de pensar el problema del otro. De esta manera, refiere a las "pruebas fenomenológicas de Max Scheler" que se encuentran en el Sympathiebuch. Particularmente relevante para este artículo es la referencia que hace Heidegger al "anexo 118 ss." También, Heidegger invita a tener presente la segunda edición del Sympathiebuch, denominada Esencias y formas de la simpatía (1923), insistiendo aquí, nuevamente, en la referencia al referido anexo, esto es (244ss.) (Heidegger, 1997:141).

La pregunta que se impone, una vez desarrollado este sucinto recorrido histórico-crítico, es ¿Qué aspectos del Sympathiebuch influyen en el pensamiento de Heidegger? Sin duda alguna, su anexo, al que me abocaré a continuación.

\section{COMENTARIO DEL ANEXO DEL SYMPATHIEBUCH}

Lo primero que llama la atención del "Anexo" (Anhang) es su sentido y finalidad ¿Por qué y para qué Scheler tiene que escribir un apéndice al Sympathiebuch? Un comentarista sostiene lo siguiente: Scheler quiso reunir en el libro los análisis de la simpatía con los análisis de la percepción del otro. Sin embargo, dado que se extendió en demasía en los análisis específicos de la simpatía, se vio obligado a desarrollar la investigación sobre la percepción del otro "in extenso" a continuación. Ello explicaría la estructuración del Sympathiebuch en una parte principal y en un Anexo. No obstante lo anterior, el Anexo goza de una relativa independencia, puesto que se vincularía con la declarada intención de Scheler de desarrollar un trabajo acerca de la teoría del conocimiento. ${ }^{9}$ Me parece que se entiende ahora mejor la crítica de Heidegger a Scheler - expuesta en el primer apartado de este trabajo - en el sentido que esas investigaciones tienen un lastre gnoseológico. Con todo, es interesante determinar qué impactó a Heidegger de ese

\footnotetext{
8 "Im selben Jahr 1913 erschien im Max Niemeyer Verlag die bedeutsame Untersuchung von Max Scheler: "Zur Phänomenologie der Sympathiegefühle und von Liebe und Hass. Mit einem Anhang über den Grund zur Annahme der Existenz des fremden Ich" (Heidegger, 2000:85).

9 Así queda estipulado en el siguiente texto póstumo de Scheler: Eine spätere Disposition (ca.1913/14) gibt einigen Aufschluss über den Zusammenhang, in den der Verfasser seine Untersuchungen zur Erkenntnislehre in der Zeit der Niederschrift von Phänomenologie und Erkenntnistheorie zu stellen plante (Scheler, 2000a:517).
} 
Anexo. La respuesta la encontraremos un poco más adelante, una vez que presente algunos momentos de tal apéndice.

En el Anexo, que lleva como subtítulo: "Acerca del fundamento de la suposición de la existencia del otro yo" (Über den Grund zur Annahme der Existenz des fremden Ich), Scheler parte de la siguiente constatación: cuando nosotros nos compadecemos, damos por supuesto en ello la existencia de otra esencia. La pregunta de Scheler es, entonces, comprensible: "¿Cómo alcanzamos la suposición de esa existencia misma?" (Scheler, 1913:118). ${ }^{10} \mathrm{O}$, formulado de otro modo "¿Qué tipo de actos son aquellos en los cuales aquel conocimiento se origina y cuáles datos son aquellos, que nosotros precisamos para tal suposición?" (Scheler, 1913:118). ${ }^{11}$ En otras palabras ¿Qué hechos de la conciencia pueden fundar la existencia del otro? Para responder estas interrogantes, Scheler analiza críticamente las teorías de su época que explican tal fenómeno, entre ellas la teoría de las "inferencias de analogía" (Analogieschlüsse) y la teoría de la "empatía" (Einfühlung). ${ }^{12}$

Puede resultar un tanto intempestivo, para el lector actual, el planteamiento de la pregunta por la existencia del otro; más aún, las referencias a una teoría de las inferencias de analogía. Con todo, cuando Scheler escribe el Sympathiebuch y su respectivo Anexo, esta teoría constituye el más dominante, reconocido y, al mismo tiempo, popular planteamiento sobre el conocimiento de lo psíquico ajeno. Tanto es así, que, como expondré luego, hasta el mismo Heidegger da cuenta de ella en Ser y Tiempo.

En relación a la teoría de la inferencia de analogía, John Stuart Mill propone que hay dos rasgos distintivos y verdaderos del ser humano: su "cuerpo" (body) y su "comportamiento" (act). De este modo, su propuesta supone que yo percibo que los otros, como yo, tienen un cuerpo; además, sé, a partir de mi propia experiencia, que mi cuerpo es la fuente de mis vivencias. Por otro lado, puedo percibir que los otros presentan un comportamiento exterior, como, a su vez, yo mismo puedo presentarlo; también sé, a partir de mi propia experiencia, que eso se produce gracias a las vivencias. En consecuencia, esta "percepción" (Wahrnehmung) permite la inferencia de los rasgos del ser humano antes mencionados.

Junto con los trabajos de Mill, es posible referir los estudios de Gustav Theodor Fechner, Hermann Paul, Ernst Mach, Alois Riehl, Benno Erdmann y H.

\footnotetext{
10 "Wie aber kommen wir zu der Annahme dieser Existenz selbst?" (Scheler, 1913:118).

11 "Welche Arten von Akten sind es, in denen jenes Wissen entspringt, und welche Gegebenheiten sind es, die wir zu solcher Annahme bedürfen?” (Scheler, 1913:118).

${ }^{12}$ Así lo sostiene Scheler: "Sowohl die Theorie, dass es 'Analogieschlüsse' seien, die dazu führen, bei Wahrnehmung von unseren Ausdruckbewegungen gleichartigen Ausdrucksbewegungen, die wir als Folgen unserer individuellen Ichtätigkeit erleben, auf gleichartige Ichtätigkeiten im Anderen zu schliessen; als die besonders von Th. Lipps vertretene Theorie, dass jene Annahme ein durch einen "Einfühlungsprozess" des Ich in die fremde Köperscheinung fundierter "Glaube" an fremde seelische Existenz sei, waren bisher Mittel, jene Schwierigkeiten zu lösen. Beide Theorien vermögen ihr Ziel indes nicht zu erreichen" (Scheler, 1913:118ss.).
} 
Driesch (Michalski, 1997:69ss.) y, en el "Anexo", Scheler formula cuatro argumentos contra la teoría de la inferencia de analogía. El primero y el tercero contra Riehl y el segundo y el cuarto contra Lipps. ${ }^{13}$ La primera crítica — citando de paso a Hume - sostiene que la creencia en existencias psíquicas ajenas existe "sin duda también para los animales, los que de seguro no hacen ninguna inferencia de analogía" (Scheler, 1913:119) ${ }^{14}$ crítica que, más tarde, será conocida por la psicología como el "argumento de la inteligencia" (Michalski, 1997:72). Lo que está discutiendo Scheler, en el fondo, es la hipótesis, según la cual un comportamiento primitivo es determinado por impulsos y sensaciones sencillos. Un contraejemplo para Scheler son los seres humanos que pertenecen a pueblos primitivos y que viven su mundo lleno de poderes y propiedades místicas.

La segunda crítica de Scheler contra la teoría de la inferencia de analogía está basada en el "argumento del espejo" (Michalski, 1997:4) que remite, a su vez, a Lipps. La observación de Scheler es la siguiente: "En segundo lugar, tenemos entonces una conciencia de nuestros movimientos de expresión - pero nosotros no pensamos en un espejo y parecido- empero, sólo en la forma de intenciones de movimiento y consecuencias de percepción de movimientos y de ubicación, mientras que nos son dadas de otras esencias ante todo sólo las imágenes ópticas de esos movimientos, cuyos datos, dados en una primera aproximación, en nada son iguales o parecidos" (Scheler, 1913:119). ${ }^{15}$ En otras palabras, Scheler critica el supuesto de la teoría de la inferencia de analogía, según el cual la percepción de los propios "movimientos de expresión" (Ausdrucksbewegungen), es semejante a los movimientos de expresión ajenos. Las inferencias de analogía no son ejecutadas normalmente sobre la base de una semejanza entre movimientos de expresión propios y ajenos, sino que, como mucho, sobre la base de una semejanza ajena con

\footnotetext{
${ }^{13}$ Theodor Lipss (1851-1914) "nació en Wallhaben (Pfalz), estudió en varias Universidades (Erlangen, Bonn, etc.); fue Privatdozent (1877-1884) y profesor extraordinario (1884-1890) en Bonn, y profesor titular en Breslau (1890-1894) y en Munich (a partir de 1894). Lipps ha sido considerado como un 'psicologista' (en el sentido por lo menos que tuvo el término 'psicologismo' en quienes, como Husserl, polemizaron contra esta tendencia). En todo caso, consideró la psicología como la ciencia filosófica fundamental o, mejor dicho, como la ciencia fundamental del espíritu, la base de toda investigación tanto de índole subjetiva como objetivo-espiritual. La psicología tiene por tema la experiencia interna, a diferencia de la experiencia externa estudiada por las ciencias naturales, pero dentro del campo de esa experiencia sitúa Lipps todos los objetos de las ciencias del espíritu y, por tanto, todos los objetos de la lógica, la estética, la historia y la filosofía misma. [...] En la ética, Lipps se aproxima decididamente al kantismo y elabora una ética de tendencia formal donde el soberano y único bien radica en la personalidad moral. En la estética se le debe la fundamentación y desarrollo de la teoría de la introafección, proyección sentimental, empatía o endopatía" (Ferrater Mora, 1999:2154ss.).

14 “"...] zweifellos auch bei Tieren, die sicher keine 'Analogieschlüsse' machen” (Scheler, 1913:119).

15 "Zweitens haben wir zwar ein Bewusstsein von unseren AUsdrucksbewegungen - aber soweit wir nicht an Spiegel denken und Ahnliches - doch nur in der Form von Bewegungsintentionen und Folgen von Bewegungs- und Lageempfindungen, während uns von anderen Wesen doch an erster Stelle nur die optischen Bilder dieser Bewegungen gegeben sind, die jenen uns gegebenen Daten zunächst in Nichts gleichen oder ähnlich sind" (Scheler, 1913:119).
} 
otro movimiento de expresión ajeno (Michalski, 1997:75ss). Ello acontece cuando uno tiene dudas respecto a que si un determinado movimiento que percibimos, llevado a cabo por alguien, se asemeja o no a un comportamiento que para uno es conocido. Piénsese, por ejemplo - dice Scheler-en el caso de un enajenado mental que parece que nos sonríe o en un primate que parece estar en una actitud reflexiva, con una mano bajo el mentón.

El tercer argumento de Scheler está relacionado con el anterior: "En tercer lugar, sin duda alguna nosotros suponemos también para tales animales la existencia de un alma, cuyos movimientos de expresión (y "acciones") en nada son iguales a los humanos, por ejemplo, para los peces y pájaros" (119). ${ }^{16}$ Scheler discute ahora con formulaciones primitivas de la teoría de la inferencia de analogía rechazando la posibilidad de comparar a los seres humanos con los animales.

Finalmente, el cuarto argumento postula que la teoría de la inferencia de analogía es lógicamente falsa, porque utiliza el concepto "yo" en dos diferentes sentidos — por un lado, en el significado de "mí yo" (mein Ich); por otro, en el significado de "un otro yo" (ein anderes Ich) - y a lo que se agrega en la inferencia otra noción de "yo". Se produce, como recalca Scheler, la falacia denominada quaterno terminorum. ${ }^{17}$

Un tema, no menor, dentro de la fenomenología es la crítica de Scheler a la "empatía" (Einfühlung). La historia de la teoría de la empatía tiene, a diferencia de la teoría de la inferencia de analogía, a la estética como protagonista principal. Se pueden mencionar en este marco los nombres de Wilhelm Perpeet, Friedrich Theodor Vischer, Robert Vischer, Theodor Lipps y Antonin Prandtls. Lipps, por ejemplo, considera que la "percepción sensorial" (sinnlische Wahrnehmung) nos proporciona "cosas" (Dinge), "objetos físicos" (physische Gegenstand), cuerpos y movimientos, los que no son interpretados necesariamente como propios de seres humanos. De ahí, considera Lipps, pertenece al ser humano la "vivencia psíquica" (seelisches Erleben), la que nunca se nos es dada mediante percepciones sensoriales, sino que mediante "percepciones internas" (innere Wahrnehmung); esto es, se nos da como vivencias propias (Michalski, 1997:81).

La "empatía" (Einfühlung) es, para sus partidarios, una característica de la naturaleza humana, que está compuesta por dos momentos: el instinto de "imitación" (Nachahmung) y el instinto de "manifestación vital" (Lebensäusserung) (Michalski, 1997:81) La empatía funciona, entonces, de la siguiente manera: yo veo como otro cuerpo se mueve de una manera determinada (por ejemplo, "él camina a zancadas") y

16 "Drittens ist es zweifellos, das wir auch bei solchen Tieren die Existenz von Beseelung annehmen, deren Ausdrucksbewegungen (und "Handlungen") den unsrigen menschlichen in Nichts gleichen, z.B. bei Fischen, Vögeln usw" (Scheler, 1913:119).

17 "Endlich futre auch tener Analogieschluss - auch wenn er geübt würde und das Material für ihn vorhanden wäre, und er überall geübt würde, wo jene Annahme besteht - auf keinen Fall zum Inhalt der in Frage stehenden Annahme. [...] Soll der Schluss ein fremdes, von meinem Ich verschiedenes Ich setzen, so ist er ein falscher Schluss, eine quaternio terminorum. Endlich beachte man den Inhalt dieser Annahme!” (Scheler, 1913:120). 
tiendo a imitarlo, es decir, me muevo de la misma manera ("yo camino a zancadas"); en esa tendencia al movimiento yace, gracias al instinto de manifestación vital, una vivencia determinada y propia. Dicho de otra manera, yo "empatizo" (einfühlen) mi vivencia con el cuerpo visto; yo, por decirlo así, "penetro" en el otro, de tal manera que lo que siento al andar a zancadas, me ayuda para saber lo que siente el otro cuando anda a zancadas. ${ }^{18}$

Por otro lado, probablemente fueron desconocidos, para Scheler, los manuscritos de Edmund Husserl sobre la "empatía" (Einfühlung) que hoy conocemos a través del segundo volumen de las Ideas. ${ }^{19}$ Con todo, uno también puede suponer que Scheler conocía perfectamente la atmósfera en la que se desarrollaron las cavilaciones de Husserl al respecto. Cuando menos, se sabe que leyó con gran avidez el trabajo de doctorado de Edith Stein, dirigido por Husserl, titulado Sobre el problema de la empatía. $^{20}$

Hechas estas aclaraciones históricas, me concentraré en la presentación de los dos argumentos de Scheler contra la teoría de la empatía, que probablemente tuvo a la vista Heidegger para hacer sus comentarios en el §26 de Ser y Tiempo. Scheler sostiene, en primer lugar, que la teoría de la empatía nos da "una hipótesis sobre el procedimiento, mediante el cual alcanzamos una suposición. Pero nunca es capaz de asegurarnos los fundamentos de esa suposición" (120). ${ }^{21}$ En otras palabras la doctrina de la empatía sería incapaz de diferenciar aquellos "círculos de casos" (Kreise von Fällen), donde involuntariamente un yo o un alma empatizan. Dicho en términos más sencillos, para Scheler, la teoría de la empatía no es capaz de separar la fuente del conocimiento de la existencia del yo ajeno de la pura empatía estética del yo, por ejemplo, en un retrato o de Hamlet, este personaje del mundo artístico, del gesto corporal de un actor. Y, a continuación, Scheler comenta agudamente: "Precisamente aquí no hay nada que decir, respecto de qué dato el proceso de la "empatía" debe coincidir con el propio yo". (120ss.). ${ }^{22}$ Uno podría decir,

${ }^{18}$ El Historisches Wörterbuch der Philosophie define la empatía de la siguiente manera: "Según Th. Lipps la empatía es la fuente del conocimiento sobre los otros, de tal modo que se desarrollan vivencias de sentimientos percibidas mediante co-ejecuciones (Mitvollzug) motoras, las que, entonces, en un acto de objetivación son atribuidas al otro como su intención o condición" (HWPh, 1972:396).

${ }^{19}$ Husserl desarrolla, de hecho, el tema de la empatía en un texto más tardío, esto es, en la V. Meditación Cartesiana.

${ }^{20}$ De este modo lo atestigua Michalski citando una carta de Edith Stein a Roman Ingarden. (1997:80).

21 "Was sie gibt, ist eine Hypothese über den Hergang, auf den es zu dieser Annahme kommt. Aber niemals vermag sie uns des Rechtes dieser Annahme zu versichern" (Scheler, 1913:120).

22 “"...] auch vermag die Theorie nicht die Einfühlung als Erkenntnisquelle der Existenz fremder Iche von der bloss ästhetischen Einfühlung des Ich zu scheiden, z.B. in ein Porträt, oder des Hamlet, dieser Person der künstlerischen Welt, in den Körpergestus eines Schauspielers. Es ist eben hier gar nicht zu sagen, auf welches Datum hin der Prozess der 'Einfühlung' des eigenen Ich stattfinden soll" (Scheler, 1913:120ss.). 
argumenta, que serían necesarios contenidos ópticos de movimientos de expresión o, por lo menos, modos de comportamiento de una esencia viva. Pero ello es, a su

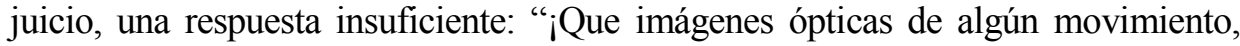
son imágenes de movimientos de expresión, es un criterio, el cual supone del mismo modo el conocimiento del fundamento de un algo ajeno y animado! $\mathrm{Su}$ interpretación como "expresión" no es el fundamento, sino que la consecuencia de esa suposición" (121). ${ }^{23}$ Con este lapidario juicio, sentencia Scheler su primera crítica a la teoría de la empatía.

La segunda es la siguiente: "Finalmente la teoría de la empatía no lleva al contenido de la suposición de la existencia de yo ajeno, a saber, de yo ajeno individual" (121ss.). ${ }^{24}$ Dicha teoría sería capaz sólo de sustentar la creencia, según la cual, mi yo yacería allí "una vez más", sin embargo, ella nunca sería capaz de sustentar este yo como ajeno u otro. En otras palabras, la teoría de la empatía sólo logra explicar la ilusión en que sucumbimos, cuando uno cree que una parte mía, de mi yo, se encuentra en el yo ajeno (Michalski, 1997:85). La objeción de Scheler apunta, entonces, a discutir el supuesto de Lipps, según el cual, el yo empático sería una "proyección" (Projektion), un "reflejo" (Spiegelung) o una especie de "radiación" (Strahlung) de mi propio yo en la aparición sensorial de un cuerpo ajeno. No hay para Scheler "duplicado" (Verdoppelung) ni "copia" (Vervielfältigung) de mi propio yo posible. A partir de lo anterior, sustenta la siguiente tesis: Sólo porque nosotros sabemos, que el yo ajeno es un individuo, "nosotros sabemos, que es un "otro"; esto no es al revés, es decir, que es un individuo, porque es un "otro" (122). ${ }^{25}$ Luego el saber de individuos ajenos incluye no sólo el saber, que ellos poseen una determinada corporalidad, sino que, sobre todo, el saber que "nosotros nunca adecuadamente podemos comprenderlos en su peculiar e individual esencia" (122). ${ }^{26}$ Emerge aquí un tópico antropológico central: el ser humano como misterio o, en categorías de Scheler, la inviolabilidad de la persona humana. ${ }^{27}$

En consecuencia, la crítica de Scheler tanto a la teoría de la inferencia de analogía como a la teoría de la empatía alcanza una conclusión negativa: Ninguna es capaz de explicar razonablemente la existencia del otro ajeno o extraño. Por ende, la alternativa de Scheler es recurrir a la fenomenología para encontrar solución a este

\footnotetext{
23 "Dass die optischen Bilder irgendwelcher Bewegungen Bilder von Ausdruckbewegungen sind, das ist eine Einsicht, welche die Kenntnis des Bestandes eines fremden beseelten Etwas eben bereits voraussetzt! Ihre Auffasung als "Ausdruck" ist nicht der Grund, sondern die Folge dieser Annahme" (Scheler, 1913:121).

24 "Endlich führt auch die Einfühlungstheorie nicht zum Inhalt jener Annahme, der Existenz fremder Iche, und zwar zu der fremder Ichindividuen" (Scheler, 1913:121ss.).

25 "[...] und nur darum wissen wir, das es ein "anderes" ist; nicht aber ist es uns ein Individuum, weil es ein "anderes" ist!" (Scheler, 1913:122).

26 “[...] dass wir diese niemals adäquat, in ihrem eigentümlichen individuellen Wesen, erfassen können" (Scheler, 1913:122).

${ }^{27}$ Así lo sostiene Scheler: "Sowohl das Ich wie der Leib findet in der erlebbaren Zugehörigkeit zur einheitlichen Person seine lezte Individualisierung” (Scheler, 1913:123).
} 
problema. "Dejémoslas aparte seriamente $\mathrm{y}$, en general, todas las suposiciones realistas y practiquemos la fenomenología pura" $(125){ }^{28}$

En este contexto fenomenológico, es posible determinar algunos tópicos que interesaron a Heidegger en su lectura del apéndice del Sympathiebuch. Ello, a partir de intuiciones propias, como de los antecedentes que aporta el texto de Michalski, quien tuvo acceso a la biblioteca personal de Heidegger y, específicamente, a las obras de Scheler.

Una primera anotación que realiza Heidegger, según Michalski, corresponde a la descripción que hace Scheler de distintos tipos de vivencias. Scheler considera que las vivencias son sencillamente "dadas" (gegeben), sin que necesariamente tengan que ser calificadas como propias o ajenas. No acontece, como supone - por ejemplo- la teoría de la empatía, que a partir de un material dado "en primera instancia" (zunächst), nuestras vivencias propias nos hayan formado imágenes de vivencias ajenas, sino que una corriente indiferente de vivencias fluye en primera instancia allí, la que contiene tanto vivencias propias como ajenas de modo inseparable y mezcladas unas con las otras; y en esa corriente se forman gradualmente firmes y configurados remolinos, los que remolcan siempre lentamente nuevos elementos de la corriente en su círculo (127). ${ }^{29}$

Según Michalski, Heidegger escribe en una nota marginal, a propósito del giro "corriente indiferente de vivencias", la siguiente expresión: "jla vida fáctica!" (das faktische Leben!) (Michalski, 1997:96), anotación que está en relación con los "Puntos a considerar para la reformulación" (Merkpunkte für die Umarbeitung) que se encuentran en la lección magistral Grundprobleme der Phänomenologie (semestre de invierno 1919/20), a los que me referí, brevemente, al comienzo de este artículo. El "acento" (Zugespitztheit) de la vida fáctica sobre el "mundo propio" (Selbstwelt), es "en el énfasis presente y aislado todavía, de una resonancia de una orientación inicial yoísticatrascendental" (Heidegger, 1993:198). ${ }^{30}$ En una orientación tal se inscriben, según Scheler lo demostró, tanto la teoría de la inferencia de analogía como la teoría de la empatía.

Una segunda observación de Heidegger está en relación al siguiente texto del Anexo del Sympathiebuch

"En primera instancia" vive el ser humano más en los otros que en sí mismo; más en la comunidad que en su propio individuo. Son una prueba de ello tanto los hechos de la vida infantil como los hechos de toda vida anímica primitiva del pueblo (127). ${ }^{31}$

\footnotetext{
28 "Lassen wir sie aber ernsthaft beiseite und überhaupt alle realistischen Voraussetzungen, und treiben wir pure Phänomenologie" (Scheler, 1913:125).

29 “[...] sondern ein indifferenter Strom der Erlebnisse fliesst "zunächst" dahin, der Eigenes und Fremdes ungeschieden und ineinandergemischt enthält; und in diesem Strome bilden sich erst allmählich fester gestaltete Wirbel, die langsam immer neue Elemente des Stromes in ihre Kreise ziehen" (Scheler, 1913:127).

30 "Zugespitztheit in der vorliegenden isolierten Betonung noch zu sehr Nachklang einer anfänglichen ichlich-transzendentalen Orientierung" (Heidegger, 1993:198).

31 "Zunächst lebt der Mensch mehr in den Anderen als in sich selbst; mehr in der Gemeinschaft als in seinem Individuum" (Scheler, 1913:127).
} 
En esta cita, por un lado, Scheler se distancia definitivamente de una concepción de la empatía al estilo de una egología monadológica, en el sentido que Husserl expondrá posteriormente en la V Meditación Cartesiana; en otras palabras, supera la concepción, según la cual, dos mónadas son las que desarrollan el proceso intersubjetivo. Para Scheler, salimos de nosotros mismos para - por decirlo asíreconocernos en el otro y ejemplifica su tesis aludiendo a las vivencias de la vida infantil, las que se construyen habitualmente en relación con los padres, el entorno, los parientes, los hermanos mayores, la ciudad natal, el país de origen, etc. Por otro lado, este pasaje del Anexo motiva la formulación del "uno" (man), un importante concepto que Heidegger desarrolla en el §27 de Ser y Tiempo. Que el ser humano, en primera instancia, no vive en sí mismo, sino que en los otros, corresponde al "uno" (man). En ese sentido, "comunidad" es sólo posible para Heidegger como una nueva ganancia a partir de la "pérdida de sí mismo" (Selbstverlorenheit) (Michalski, 1997:99).

Por último, cabe mencionar que Heidegger evalúa positivamente no sólo la tematización scheleriana de las vivencias y de las relaciones de vivencias, sino también los análisis sobre lo anímico y lo corporal. ${ }^{32}$

\section{REPERCUSIONES QUE EL ANEXO DEL SYMPATHIEBUCH TUVO EN SER Y TIEMPO}

La problemática de la intersubjetividad en el pensamiento de Heidegger no es una cuestión obvia sino, más bien, controversial. ${ }^{33}$ La desarrolla incipientemente en el Capítulo Cuarto de la Primera Sección de Ser y Tiempo cuya introducción se inicia con una síntesis de los logros preliminares alcanzados por Heidegger y con una proyección de los mismos. Así, respecto al análisis de la mundaneidad del mundo, sostiene que tuvo permanentemente ante su mirada el fenómeno del estar-en-el-mundo en su totalidad, "sin que por ello se destacaran todos sus momentos constitutivos con la misma claridad fenoménica que el fenómeno del mundo mismo" (1997:139). En otras palabras el análisis de los entes intramundanos y del Dasein no ha agotado todos los fenómenos por describir. Por ejemplo, no se ha aclarado aún “¿Quién es el Dasein en la cotidianidad?” (139).

${ }^{32}$ Así lo sostiene, por ejemplo, Heidegger: "Vivencias, relaciones de vivencias, sentido de la existencia, y realidad de los actos de las vivencias-Persona (Cfr., jexcelente en Scheler!)" (Heidegger, 1993:161). "Erleben, Erlebniszusammenhang, Sinn der Existenz und Wirklichkeit der Erlebnisakte-Person (vgl. Ausgezeichnetes bei Scheler!)" (Heidegger, 1993:161).

${ }^{33}$ Sigo aquí la interesante tesis de Alejandro G. Vigo expuesta en el artículo "Identidad, decisión y verdad. Heidegger, en torno a la constitución del "nosotros"', (2008).

${ }^{34}$ Es interesante detenerse un momento en la pregunta que formula Heidegger: ¿Quién es el Dasein? Uno esperaría, dada la tradición antropológica, que la pregunta fuera ¿Qué es el Dasein?, equiparando dicha interrogante con la pregunta central de la antropología filosófica, esto es ¿Qué es el hombre? Precisamente, ésa no es la intención de Heidegger. En esta ocasión, la crítica a la antropología filosófica es que ésta ha formulado mal la pregunta por la existencia humana o, en palabras de Heidegger, se hace necesario "un planteamiento correcto de la pregunta" (Heidegger, 1997:139), puesto que ha quedado encerrada en marcos propios de la "ontología del estar-ahí" (Vorhandenheitontologie). Esta temática es abordada nuevamente por Heidegger, más tarde, en la conocida Carta sobre el Humanismo (2001:259-297). 
Dicho de otra manera, a Heidegger le interesa explicar cuál es la identidad que le corresponde al ente que somos nosotros mismos, sin por ello caer en un análisis de corte antropológico. El esclarecimiento de dicha interrogante conduce a estructuras que son cooriginarias con el estar-en-el-mundo, es decir, "el coestar (Mitsein) y la coexistencia (Mitdasein)" (139). Por último, otro tópico que toca Heidegger en este capítulo, tiene que ver con aquel modo de ser que funda el modo cotidiano de ser-sí-mismo, esto es, el "se" o el "uno" (das Man)" (139).

De este modo, en el parágrafo $\$ 25$ de Ser y Tiempo, Heidegger despliega la pregunta por "quién es el Dasein". Tal parece que esa pregunta ya fue respondida en el parágrafo $\S$, cuando abordó el tema de las determinaciones fundamentales del Dasein, o sea, su "tener-que-ser" (Zu-sein) y su "ser-cada-vez-mío" (Jemeinigkeit). Con todo, a un nivel óntico, quedó sólo bosquejado que este ente correspondería a un yo. Así, "el quién queda respondido desde el "yo mismo", el "sujeto", el "sí" (das Selbst)." (140) Con esta afirmación, Heidegger complementa la discusión planteada en el §10 donde delimita la analítica existencial de algunas ciencias particulares y critica duramente la "antropología tradicional" — conformada por la definición griega del hombre, la antropología teológica cristiana y la antropología moderna - porque ha dejado en el olvido la "pregunta por su ser" (74). Heidegger profundiza su crítica a la antropología moderna, en especial, a su concepto central: el sujeto. En sus palabras, el mencionado "sí" (das Selbst) ha sido interpretado ontológicamente ${ }^{35}$ como una "región cerrada" o "subyacente", es decir, "como el subiectum" (140). Habría que agregar que el "sí" ha sido erróneamente interpretado de esa manera porque, incluso, en aquellas perspectivas que interpretan al "yo" más allá de la sustancia del alma, de la cosidad de la conciencia $^{36}$ o del carácter de objeto de la persona — en directa alusión a Scheler"ontológicamente se sigue planteando algo cuyo ser conserva de un modo expreso o tácito el sentido del estar-ahí" (140). En último término, dichas perspectivas mantienen el lastre de la sustancialidad o de la ontología del "estar-ahí" (Vorhandenheit). En otras palabras, Heidegger procura hacer presente que el "si" no debe ser entendido cósicamente, como algo dado, sino que debe dejar espacio incluso para un "no yo", para "la perdida de sí" (Seinsverlorenheit), para el abandono de sí mismo.

Es en este marco que Heidegger alude positivamente al pensamiento de Scheler: No es posible pensar el "yo" o el "si'" únicamente de una manera individual, sino que abierto a los otros o a los prójimos

La aclaración del estar-en-el-mundo ha mostrado que no "hay" inmediatamente, ni jamás está dado un mero sujeto sin mundo. Y de igual modo, en definitiva, tampoco se da en forma inmediata un yo aislado sin los otros. Pero si "los otros" ya están siempre co-existiendo en el estar-en-el-mundo, esta constatación fenoménica no

\footnotetext{
${ }^{35}$ Hay que precisar que el término "ontológico" alude aquí a la ontología del estar-ahí y no a la ontología fundamental.

${ }^{36}$ Según Theunissen, en alusión a Husserl. Cfr. M. Theunissen, (1965:157).
} 
debe inducirnos a considerar la estructura ontológica de lo así "dado" como algo obvio y no necesitado de mayor investigación (142).

Heidegger reitera, aquí, una de sus primeras conclusiones preliminares: el análisis del estar-en-el-mundo demostró que no hay sujeto sin mundo. Al mismo tiempo, inspirándose explícitamente en los análisis de Scheler, sostiene que dicho sujeto no puede ser calificado como solipsista; muy por el contrario, el Dasein está siempre vinculado con otros Dasein (Vigo, 2008:237). De esta manera, la tesis heideggeriana, según la cual los prójimos están siempre coexistiendo en el estar-en-el-mundo, es fundada por Heidegger - como ya sostuve en la primera parte de este trabajo - tanto en los análisis del Anexo del Sympathiebuch como en la lectura del apéndice de Esencia y formas de la simpatía. El punto por dilucidar es la manera en que se da dicha coexistencia.

Por otro lado, es posible rastrear, nuevamente, la influencia del pensamiento de Scheler en el parágrafo \$26 de Ser y Tiempo donde Heidegger se refiere la teoría de la empatía. Heidegger sostiene que la descripción del mundo circundante inmediato, por ejemplo, del mundo en que trabaja el artesano, "nos hizo ver que con el útil que se está elaborando comparecen "también" los otros, aquellos para quienes la "obra" está destinada" (143). Dicha comparecencia se manifiesta a través de la remisión de dicho útil. Por ejemplo, el maletín que uso ha sido comprado en una determinada tienda atendida por su propia dueña, me fue regalado por mis padres y en éste guardo los apuntes con que enseño a mis alumnos. Estos "otros", sostiene Heidegger, comparecen en el contexto de útiles a la mano en el mundo circundante y "no son añadidos por el pensamiento a una cosa que inmediatamente sólo estuviera-ahí" (143). Hay que destacar en esta cita la referencia a los otros como algo "no añadido". Heidegger rechaza, entonces - me parece a partir de los estudios schelerianos - los planteamientos de las teorías de la inferencia de analogía o de la empatía. Para él, es innecesario preguntarse si la percepción de los propios movimientos de expresión es semejante a los movimientos de expresión ajenos o si "penetro" en el otro, de tal manera que lo que yo siento es lo mismo que el otro siente. En definitiva, resulta ser un contrasentido imaginarse la existencia de los otros, porque ellos habitan el mundo junto a mí, incluso, cuando estoy solo.

No obstante lo anterior, Heidegger refuerza su interpretación del otro, demostrando a mi juicio, el peso que teorías como la de la empatía tenían en su época. Él se pregunta ¿No empieza también ella [la caracterización del comparecer de los otros] destacando y aislando al "yo" de tal manera que luego será necesario buscar una vía para pasar desde este sujeto aislado hacia los otros? (143. El agregado es mío). En otras palabras ¿No estará incurriendo su planteamiento en el mismo malentendido que la teoría de la inferencia de analogía o de la empatía? Heidegger considera que no. Para evitar este malentendido será necesario, sostiene, considerar en qué sentido se habla de los otros. "Los otros no quiere decir todos los demás fuera de mí, y en contraste con el yo; los otros son, más bien, aquellos de quienes uno mismos generalmente no se distingue, entre los cuales también se está" (143). Nuevamente el argumento de Heidegger se diferencia de teorías que suponen que el otro es distinto de mí. Más 
bien, uno tiene una relación irrenunciable con los otros, con los que se confunde. Rechaza, en consecuencia, las explicaciones teóricamente elucubradas que se nos imponen para dar cuenta del estar-ahí de los otros, ateniéndose firmemente al dato fenoménico que ha mostrado su comparecer en el mundo circundante. De paso, prepara el escenario para tratar el tema del "uno" o del "se". Con todo, previo a su desarrollo, Heidegger alcanza otra conclusión relevante: en virtud de este estar-enel-mundo determinado por el "con", el mundo es desde siempre el que yo comparto con los otros. El mundo del Dasein es un mundo en común (Mitwelt). El estar-en es un coestar (Mitsein) con los otros. El ser-en-sí intramundano de éstos es la coexistencia (Mitdasein) (144).

En esta referencia aparecen dos de los temas centrales del parágrafo §26 de Ser y Tiempo, esto es, el coestar y la coexistencia. Ellos deben ser, en cualquier caso, diferenciados. El coestar es una estructura existencial del Dasein, según la cual todo Dasein individual está con otros en un mismo mundo. En cambio, la coexistencia es el modo como son los otros Dasein, que son convivientes en este mundo; es el Dasein de los demás. En consecuencia, la prioridad entre el coestar y la coexistencia la tiene el primero, ya que dicha coexistencia se funda en el coestar. ${ }^{37}$ En palabras de Heidegger, "el Dasein propio sólo es coexistencia en la medida en que, teniendo la estructura esencial del coestar, comparece para otros" (146).

Por otro lado, luego de vincular el coestar con otras estructuras del Dasein como por ejemplo, el "cuidado" (Sorge), la "solicitud" (Fürsorge) —que serían los modos de ser del coestar (Theunissen, 1965:164) - y la comprensión de otros, Heidegger toma distancia explícitamente de la teoría de la empatía. ${ }^{38}$ Lleva a cabo lo anterior, a partir del siguiente problema: la comprensión de la vida psíquica ajena. Este fenómeno, llamado, de manera no precisamente feliz "Einfühlung" (empatía, endopatía), debería — en cierto modo, por primera vez - tender ontológicamente el puente desde el propio sujeto, dado primeramente solo, hacia el otro sujeto, que empezaría por estar enteramente cerrado (149).

La relación con los otros se convierte, entonces, en la proyección "a otro" del propio ser, lo que Scheler criticaba como imitación o espejeo en el otro; es decir, el otro es un doblete de sí mismo. Naturalmente, este punto de vista, propio de la teoría de la empatía, es rechazado por Heidegger quien identifica una base endeble en la argumentación. Su supuesto no es válido, a saber: "que el estar vuelto del Dasein hacia sí mismo es equivalente al estar vuelto hacia otro" (149). Más bien, la situación es a la inversa; es decir, no es la "empatía" la que constituye el coestar, sino que ella

37 Más adelante Heidegger agrega sobre el coestar: "Esta coexistencia de los otros queda intramundanamente abierta para un Dasein y así también para los coexistentes, tan sólo porque el Dasein es en sí mismo esencialmente coestar (Mitsein). La afirmación fenomenológica: el Dasein es esencialmente coestar, tiene un sentido ontológico-existencial. (...) El coestar determina existencialmente al Dasein, incluso cuando no hay otro que esté fácticamente ahí y que sea percibido. También el estar solo del Dasein es un coestar en el mundo" (Heidegger, 1997:145).

${ }^{38}$ Para Theunissen, Heidegger tiene como contraparte en esta crítica a Husserl (1965:165). 
es posible tan sólo sobre la base de éste. La empatía, por ende, no puede ser entendida como un fenómeno existencial originario.

Finalmente, como ya sostuve, es posible identificar vestigios del pensamiento de Scheler en el parágrafo \$27 de Ser y Tiempo, particularmente en la elaboración del concepto del "uno" o del "se" ( $m a n$ ), pero no entraré en el detalle de la discusión, sino que presentaré algunas implicancias.

Como mostré al inicio de este apartado, si Heidegger se pregunta por el “quién" del Dasein, en este parágrafo responde dicha interrogante: “El 'quién' es el impersonal, el 'se' o el 'uno' (das Man)" (151). En otras palabras, lo que llama "das Man" es el sujeto impersonal de frases tales como "se dice", "se cuenta", "se hace"; es un fenómeno delegatorio, es un colectivo y un dato originario. Desde ahí, desde esta impersonalidad, surge y es pensable el "yo", el "tú" y el "nosotros". Y es aquí donde resuenan las palabras de Scheler, según las cuales uno vive más en los otros que en sí mismo; más en la comunidad que en su propio individuo. En consecuencia, la relación entre Scheler y Heidegger es mucho más que un conjunto de referencias y lecturas cruzadas. Es, más bien, una historia de influencias poco atendidas.

\section{Universidad Católica del Maule* Facultad de Ciencias Religiosas y Filosóficas Departamento de Filosofía Casilla 617. Talca (Chile) enmunoz@ucm.cl}

\section{BIBLIOGRAFÍA}

AA. VV. Historisches Wörterbuch der Philosophie. Joachim Ritter (Ed.). Vol. II, Stuttgart:

Schwabe \& Co., 1972.

Ferrater Mora, José. Diccionario de Filosofía. Barcelona: Ariel, 1999.

Heidegger, Martin. Introducción a la fenomenología de la religión. Traducido por Jorge Uscatescu. México: F.C.E. / Siruela, 2006.

------ Zur Sache des Denkens. Tübingen: Max Niemeyer, 2000.

------ Ser y Tiempo. Traducido por Jorge Eduardo Rivera. Santiago: Universitaria, 1997.

------ Grundprobleme der Phänomenologie. Editado por Hans-Helmuth Gander. Frankfurt: Vittorio Klostermann, 1993.

------ Hitos. Madrid: Alianza, 2001.

Husserl, Edmund. Ideas relativas a una fenomenología pura y una filosofía fenomenológica. Libro Segundo: Investigaciones fenomenológicas sobre la constitución. México: F.C.E., 2005.

Jaspers, Karl. Psychologie der Weltanschauung, Springer, 4. Edición. Berlin-GöttingenHeidelberg, 1954.

Michalski, Michael. Fremdwahrnehmung und Mitsein. Zur Grundlegung der Sozialphilosphie im Denken Max Schelers und Martin Heideggers. Bonn: Bouvier, 1997.

Scheler, Max. Der Formalismus in der Ethik und die materiale Wertethik. Editado por Manfred S. Frings. Bonn: Bouvier, 2000. 
------ Schriften aus dem Nachlass. Editado por Manfred S. Frings, Vol. I, Zur Ethik und Erkenntnislehre. Bonn: Bouvier, 2000.

------ Esencia y formas de la simpatía. Salamanca: Sígueme, 2005.

------ Zur Phänomenologie und Theorie der Sympathiegefühle und von Liebe und Hass. Mit einem Anhang über den Grund zur Annahme der Existenz des fremdes Ich. Halle a.S.: Max Niemeyer, 1913.

Smith, Adam. La teoría de los sentimientos morales. Traducido por Carlos Rodríguez Braun. Madrid: Alianza, 1997.

Theunissen, Michael. Der Andere. Studien zur Sozialontologie der Gegenwart. Berlin: Walter de Gruyter, 1965.

Vigo, Alejandro. "Identidad, decisión y verdad. Heidegger, en torno a la constitución del "nosotros"', en Arqueología y aleteiología y otros estudios heideggerianos. Buenos Aires: Biblos, 2008:222-249. 